\title{
Skin impedance is not a factor in transcutaneous electrical nerve stimulation effectiveness
}

This article was published in the following Dove Press journal:

Journal of Pain Research

19 August 2015

Number of times this article has been viewed

\author{
Carol GT Vance' \\ Barbara A Rakel ${ }^{1,2}$ \\ Dana L Dailey' \\ Kathleen A Sluka ${ }^{1,2}$ \\ 'Department of Physical Therapy and \\ Rehabilitation Science, University of \\ lowa Carver College of Medicine, \\ 'University of lowa, College Nursing \\ lowa City, IA, USA
}

Objective: Transcutaneous electrical nerve stimulation (TENS) is a nonpharmacological intervention used to manage pain using skin surface electrodes. Optimal electrode placement is unclear. We hypothesized that better analgesia would occur if electrodes were placed over sites with lower skin impedance. Optimal site selection (OSS) and sham site selection (SSS) electrode sites on the forearm were identified using a standard clinical technique.

Methods: Experiment 1 measured skin impedance in the forearm at OSS and SSS. Experiment 2 was a crossover design double-blind randomized controlled trial comparing OSS-TENS, SSS-TENS, and placebo TENS (P-TENS) to confirm differences in skin impedance between OSS and SSS, and measure change in pressure pain threshold (PPT) following a 30-minute TENS treatment. Healthy volunteers were recruited (ten for Experiment 1 [five male, five female] and 24 for Experiment 2 [12 male, 12 female]). TENS was applied for 30 minutes at $100 \mathrm{~Hz}$ frequency, $100 \mu$ s pulse duration, and "strong but nonpainful" amplitude.

Results: Experiment 1 results demonstrate significantly higher impedance at SSS (17.69 $\pm 1.24 \Omega)$ compared to OSS $(13.53 \pm 0.57 \Omega)(P=0.007)$. For Experiment 2, electrode site impedance was significantly higher over SSS, with both the impedance meter $(P=0.001)$ and the TENS unit $(P=0.012)$ compared to OSS. PPT change was significantly greater for both OSS-TENS $(P=0.024)$ and SSS-TENS $(P=0.025)$ when compared to P-TENS. PPT did not differ between the two active TENS treatments $(P=0.81)$.

Conclusion: Skin impedance is lower at sites characterized as optimal using the described technique of electrode site selection. When TENS is applied at adequate intensities, skin impedance is not a factor in attainment of hypoalgesia of the forearm in healthy subjects. Further investigation should include testing in patients presenting with painful conditions.

Keywords: transcutaneous electrical nerve stimulation, pain, impedance, electrode site selection

\section{Introduction}

Transcutaneous electrical nerve stimulation (TENS) is a noninvasive intervention used in rehabilitation to modulate pain. However, there is conflicting literature on the effectiveness of TENS. Eight Cochrane reviews of TENS and painful conditions from 2008 to 2014 end with a determination of "inconclusive", "insufficient", or "conflicting evidence". ${ }^{1-8}$ One factor that may contribute to this conflict is the use of appropriate parameters of application. Negative findings in TENS reviews may be explained by low fidelity, with bias in treatment outcome measures and suboptimal dosing. ${ }^{9}$ One possible influence on TENS efficacy for which little data exist is the modifiable parameter of electrode placement sites. Textbooks describe multiple methods of electrode placement and include segmental, peripheral, or contralateral site placement. A method that has
Correspondence: Carol GT Vance Department of Physical Therapy and Rehabilitation Science, University of lowa Carver College of Medicine, 500 Newton Road, I-252 Medical Education Building, lowa City, IA 52242, USA

$\mathrm{Tel}+$ I 319335 979|

Fax +l 3193359707

Email carol-vance@uiowa.edu 
been used clinically is the use of "sites of least resistance" for application of TENS. Using this technique, the therapist detects points of decreased impedance - these points are associated with peripheral nerve anatomy and acupuncture points. ${ }^{10-15}$ For the purpose of this paper, this procedure will be referred to as optimal site selection (OSS).

Clinically, application of TENS at acupoint sites (considered low resistance sites) reduces pain and may be more effective than when applied over nonacupoint sites. ${ }^{16}$ In healthy controls, electroacupuncture (EA) over acupoint sites significantly increases heat pain thresholds from baseline, ${ }^{17}$ while TENS and EA significantly increase pressure pain thresholds (PPTs). ${ }^{18-22}$ TENS applied at acupoints decreases pain following laparoscopic surgery, ${ }^{23}$ chronic prostatitis/ pelvic pain syndrome, ${ }^{24}$ primary dysmenorrhea, ${ }^{25}$ total hip arthroplasty, ${ }^{26}$ and post-operative sinusotomy ${ }^{27}$ when compared to sham TENS. More importantly, TENS at acupoint sites has been shown to be associated with reduced opioid intake, nausea, and dizziness when compared to TENS at nonacupoint sites in postoperative hysterectomy patients. ${ }^{16}$ Although these studies show that TENS over acupoints is effective, it is not clear how these sites are selected and if they are actually associated with lower impedance. Acupoints are thought to be sites with lower skin impedance. ${ }^{28-30}$ Thus, using the method of OSS may be an ideal method for finding acupoint sites.

The purpose of these experiments was to 1) determine if points of least resistance, determined with a common clinical technique, showed lower impedance and 2) determine if electrodes placed over points of least resistance (OSS) showed greater analgesia than electrodes placed over sham sites (SSS, sham site selection). We therefore performed two experiments. The first experiment determined if impedance was lower over points of least resistance (OSS) when compared to SSS. The second experiment determined PPT before and after active TENS placed over OSS and SSS when compared to placebo TENS (P-TENS). We further confirmed that selection sites had lower impedance and examined if active TENS produced its effects with lower intensity and more comfort when electrodes were placed over OSS.

\section{Methods}

Both experiments were approved by the Institutional Human Subjects Review Board at the University of Iowa. The first experiment evaluated skin impedance over eight sites in the forearm in healthy controls, characterized as either OSS or SSS during a single visit. The second experiment was a crossover, double-blind randomized clinical trial, with subjects randomly allocated to one of six orders of intervention (TENS with OSS, TENS with SSS, or P-TENS with OSS). Subjects completed three visits, receiving a different allocation each visit. Outcome measurements were taken before and during a single TENS treatment. Skin impedance was detected over the OSS and SSS with two different techniques. The devices were FDA (US Food and Drug Administration) approved for the indicated usage in the United States.

\section{Subjects}

Ten healthy subjects (five male, five female) were recruited for Experiment 1, and 24 healthy subjects (12 female, 12 male) were recruited for Experiment 2. Inclusion criteria for both experiments were being between 18 and 60 years of age with no current pain condition, willingness to avoid physical exercise 4 hours prior to testing, and having urine specific gravity (USG) in the range of 1.000-1.200 (to ensure adequate hydration). USG was determined using test strips (Teco Diagnostics, Anaheim, CA, USA) from a $\sim 5 \mathrm{~mL}$ clean catch urine sample. Subjects who tested in the normal USG range of 1.000-1.020 were allowed to proceed to further testing. ${ }^{31-33}$ This screen was included to avoid differences in hydration status as previous studies indicated that hydration affects impedance. ${ }^{29,30,34,35}$ Exclusion criteria included recent trauma or loss of sensation in right upper extremity, currently taking pain medication, current acute or chronic pain, pregnancy, cardiac pacemaker, stroke, myocardial infarction, or other serious pathology for both experiments. Prior TENS use was used as an exclusion criteria for Experiment 2. The sequentially numbered, opaque, sealed envelopes (SNOSE) allocation concealment protocol randomized subjects to the treatment order for the TENS trial (Experiment 2). ${ }^{36}$ The allocation envelopes served to dictate the order in which the three treatments were allocated. Separate envelopes were developed for male and female subjects.

\section{Classification of OSS and SSS}

The technique for site classification in both experiments proceeded as follows: with TENS parameters set at $50 \mathrm{~Hz}$, $100 \mu \mathrm{s}$, continuous mode, and electrode gel covering the surface of each $2 \times 2$ inch carbon electrodes, the assessor held one gelled electrode in the palm of her right hand with the subject doing the same with the other electrode. The assessor moistened the tip of her right index finger and made contact over the dorsum of the hand on the test side. The level of pressure was maintained such that the pad of the index finger is in contact with the subject's skin without blanching of the assessor's nail hyponychium. TENS amplitude was 
increased until sensory tingling was felt in the pad of the index finger. The assessor glided her finger over the optimal site at the base of the second metacarpal and determined the lowest intensity which differentiated this point from the area surrounding it. The assessor held that minimal amplitude constant and evaluated the sham site in the midsection on the lateral border of the fifth metacarpal to verify that no electrical sensation was present. This selection technique was used in both experiments. The protocol is extended for each experiment in the following sections.

\section{Outcome measures Impedance}

An impedance meter (Checktrode 1089e, UFI, Morro Bay, CA, USA) was used to measure impedance between electrode pairs in both experiments. The unit test frequency was $30 \mathrm{~Hz}$ and displayed impedance in Ohms. Three readings were taken, and an average value was used in analysis.

\section{Experiment I}

Eight distinct areas of the dorsal aspect of the forearm were identified and marked using a flexible plastic grid with eight $5 \times 5 \mathrm{~cm}$ sections. Two additional sites were identified; one at the base of the first and second metacarpal bones in the thenar eminence (optimal site) and the other at the lateral aspect of the hand bisecting the fifth metacarpal (sham site) (Figure 1A). Impedance was measured at each of the eight forearm sites when paired with the optimal site and with the sham site on the hand (Checktrode 1089e, UFI). Electrodes were then removed, and a second assessor used the OSS technique to assess each of the eight areas on the forearm to classify each area as either an SSS or an OSS. An OSS was identified when a definite electrical sensation was present in the fingertip, and an SSS was identified if there was minimal or no sensation. For odd-numbered subjects, impedance was measured as outlined above, and for even-numbered subjects, the protocol was reversed with regard to the impedance meter and site classification.

\section{Experiment 2}

Four electrode sites (2 sets of 2) were located and marked according to the site classification protocol. One OSS and one SSS were identified just below the elbow, and one OSS and one SSS were identified in the hand (Figure 1B). The electrode impedance meter (Checktrode 1089e, UFI) was used to measure the impedance through the two electrodes placed on the OSS sites and between electrodes placed on the two SSS sites. The electrodes remained in place, and

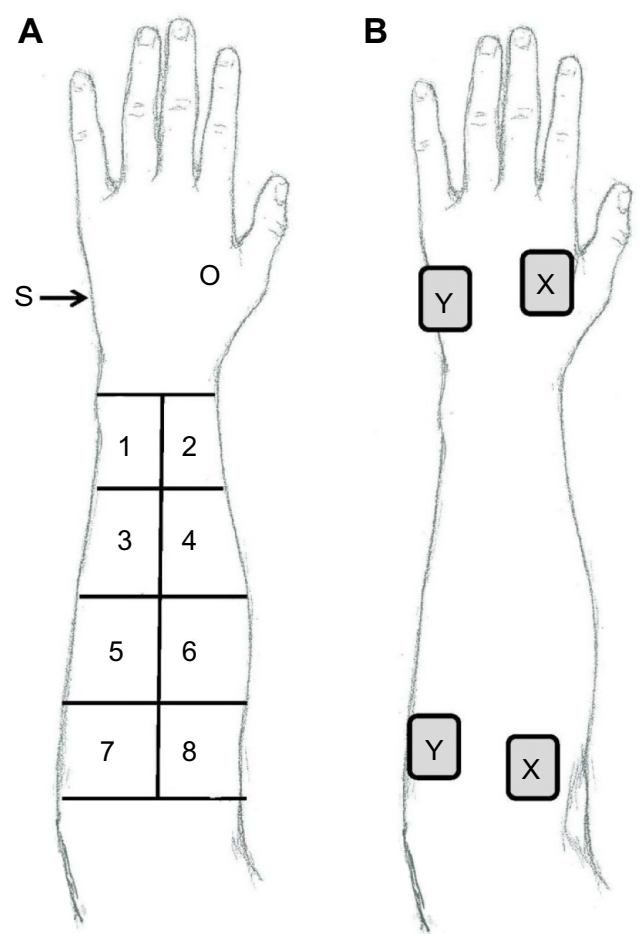

Figure I Measurement of impedance between electrode pairs in Experiments I and 2 .

Notes: (A) Diagram depicting sites used in classification of impedance of the forearm in Experiment I. (B) Sample of electrode placement for Experiment 2 where sites marked " $X$ " represent OSS and those marked " $Y$ " represent SSS. Abbreviations: $\mathrm{O}$, optimal site; $\mathrm{S}$, sham site; OSS, optimal site selection; SSS, sham site selection.

the instrumentation was changed to measure voltage with an AC current source. The Rehabilicare Maxima TENS unit (DJO Global, Vista, CA, USA) with pulse duration of $100 \mu$ s and pulse frequency of $100 \mathrm{~Hz}$ was placed in series with the patient, and voltage measures were taken from an oscilloscope (Hitachi V-1565, Hitachi Denshi, Ltd, Tokyo, Japan). Voltage was derived from the peak-to-peak values at 3, 6, 9, and $12 \mathrm{~mA}$. Impedance was calculated using measured voltage $(V)$, current $(I)$ values from prior TENS calibration, with the formula: impedance $(Z)=V / I$.

\section{Pressure pain threshold}

PPT was used as the primary outcome measure for effects of TENS in Experiment 2. A handheld digital pressure algometer (Somedic AB, Farsta, Sweden) with a $1 \mathrm{~cm}^{2}$ circular probe was used to apply pressure perpendicular to the skin at $50 \mathrm{kPa} / \mathrm{s}$. Three PPT recording sites were marked 8, 9, and $10 \mathrm{~cm}$ below the lateral elbow flexion fold over the extensor mass. Subjects were given a handheld switch to press to indicate when the pressure applied would be described as pain (distinct from pressure or discomfort). ${ }^{37}$ Subjects were instructed to consider a scale of $0-10$, where 0 is "no pain" and 10 is "worst pain imaginable", and to press the button 
when they considered the sensation to be a 1 out of 10 . The importance of replicating their method of assessment for each trial was stressed. PPT was recorded at baseline, 15, and 30 minutes. Two measurements were taken at each of the recording sites at each time point, and an average of all trials was used in data analyses. A difference score between baseline and 30 minutes and area under the curve (AUC) from baseline to 30 minutes was used in statistical analyses.

\section{Subject-perceived comfort}

A $10 \mathrm{~cm}$ visual analog scale was used to assess subject comfort during the TENS application in Experiment 2. Subjects were advised to rate their level of comfort during the TENS application by marking a single vertical line on the $10 \mathrm{~cm}$ visual analog scale, with anchors of 0 "most comfortable" to 10 "most uncomfortable". Scores ranged from 0 to 10 , with lower numbers representing more treatment comfort, and higher numbers indicating that the treatment was more uncomfortable.

\section{TENS application}

In Experiment 2, TENS was administered with the Rehabilicare Maxima TENS unit (DJO Global) by a trained allocator who was not involved in data collection. EMPI 5000 electrodes (DJO Global) were used for all subjects. The active unit delivered an asymmetrical square waveform with pulse duration of $100 \mu \mathrm{s}$ and pulse frequency of $100 \mathrm{~Hz}$. The previously validated placebo unit ${ }^{38}$ was identical in settings and appearance to the active unit; the difference being it was active only during the intensity adjustment period and slowly ramped down to "off" in 45 seconds.

A standardized script was read, and intensity was increased to sensory threshold and then to maximally tolerated intensity. We previously showed that higher intensities of stimulation increased analgesia; ${ }^{39}$ therefore, for the active TENS intervention, the intensity was adjusted every 5 minutes throughout the 30 -minute treatment as determined by the subjects' answers to the following questions: 1) Are you doing alright? 2) May I turn it up? For subjects receiving the placebo treatment, the intensity was advanced to a comfortable sensory level, and every 5 minutes, subjects were asked "Are you doing alright"? Stimulus amplitude was not adjusted during the 5-minute checks as the intensity had ramped to zero. The unit display of the original sensory amplitude remained throughout the entire treatment and was recorded in $\mathrm{mA}$ every 5 minutes, and this value was used in data analysis. TENS was applied for 30 minutes.

\section{Blinding}

The outcome assessor was blinded to intervention group by the use of an independent TENS allocator, the allocation concealment protocol, and a pouch concealing the TENS unit during treatment. In addition, because all four electrodes were placed at each visit with lead wires leading to the concealed TENS unit, the assessor was blinded to which electrode pair received the active stimulation. If muscular contractions were present, TENS intensity was decreased until no visible contraction was present by the TENS allocator just prior to the return of PPT assessor. To facilitate subject blinding, all four electrode sites were marked on each subject with no specific information given to subjects with regard to the difference between the sites. To assess investigator blinding the outcome, assessor was asked the following questions at the conclusion of the TENS application: 1) Do you feel the TENS application was active, placebo, or I don't know? 2) Do you feel the sites were optimal, sham, or I don't know?

\section{Protocol}

\section{Experiment I}

Once informed consent was obtained, demographic information and a urine specimen were collected to screen for hydration. After meeting the inclusion criteria for hydration, height and weight were recorded, and subjects were placed in the seated position with the right forearm resting on a table. The dorsal surface of the forearm and the palmar surface of the hand were cleansed. Impedance of the forearm was then performed at eight areas to evaluate the electrode site classification technique.

\section{Experiment 2}

Once informed consent was obtained, demographic information and a urine specimen were collected to screen for hydration. After meeting the inclusion criteria for hydration, height and weight were recorded, and subjects were then randomly allocated to one of six orders of TENS allocation (OSS-TENS, SSS-TENS, P-TENS). Subjects completed three visits, receiving a different allocation each visit. At all three visits, the dorsal surface of the forearm as well as the palmar surface of the hand were cleansed, and impedance at the electrode sites and PPTs were measured prior to application of TENS or P-TENS. PPTs were measured 15 and 30 minutes after the start of TENS or P-TENS. Patient comfort was measured at the end of each visit. TENS intensity was recorded every 5 minutes. Assessor blinding was measured at the completion of each visit. 


\section{Data analysis}

Experiment I

The mean and standard errors were calculated for demographics and impedance measures for each of the eight areas. The impedance values were assigned to OSS or SSS as determined by the site classification technique. Differences in impedance between SSS and OSS sites were analyzed using a paired $t$-test.

\section{Experiment 2}

Sample size calculations were made using a mean difference of $11.97 \mathrm{kPa}$ and a standard deviation of $12.63 \mathrm{kPa}$ obtained from data collection involving two active TENS treatments. ${ }^{35}$ With significance level of $P<0.05$ and power of $80 \%$, sample size needed was 24 per treatment. PPT change scores were calculated by subtraction of baseline values from the 15- and 30-minute measurements. Descriptive statistics were calculated for each variable. Normality was evaluated using the Kolmogorov-Smirnov test. PPT values were converted to AUC during TENS treatment. Repeated measures analysis of variance (ANOVA) was performed on baseline PPT, impedance measures, change in PPT and AUC between treatments, and followed with a paired $t$-test post hoc analysis. The impedance scores for each of the four intensities (3, 6, 9, and $12 \mathrm{~mA})$ were analyzed using repeated measures ANOVA and were also averaged and analyzed for overall differences. Repeated measures ANOVA were also performed on intensity and perceived comfort. Follow-up paired $t$-tests were performed to test significant differences between visits. Statistical significance was considered at $P<0.05$.

\section{Results}

\section{Experiment I}

All ten patients completed the testing (Table 1) for subject demographics, descriptive and impedance measures. Impedance measured $13.53 \pm 0.57 \Omega$ for areas on the forearm classified as OSS. Areas classified as SSS had a mean impedance of $17.69 \pm 1.24 \Omega$ and were significantly higher than sites classified as OSS $(P=0.007)$.

\section{Experiment 2}

Figure 2 provides the consort diagram for the current randomized controlled trial. A total of 24 subjects were allocated to a treatment order and completed the testing. A description of the room (room temperature and humidity), demographic information, subject information (USG, impedance, maximum intensity), and baseline PPT measures for Experiment 2
Table I Demographics and impedance measures for Experiment I for comparison of sham and optimal sites

\begin{tabular}{ll}
\hline Variable & $\mathbf{N}=\mathbf{1 0}$ \\
\hline Age (year) & $23.3 \pm 4.4$ \\
Sex & $5 \mathrm{M}, 5 \mathrm{~F}$ \\
BMI & $23.3 \pm 3.4$ \\
USG & $1.01 \pm 0.004$ \\
Room temperature $\left({ }^{\circ} \mathrm{F}\right)$ & $73.1 \pm 2.2$ \\
Room humidity $(\%)$ & $48.8 \pm 1.8$ \\
Impedance optimal sites $(\Omega)$ & $13.53 \pm 0.57^{*}$ \\
Impedance sham sites $(\Omega)$ & $17.69 \pm 1.24$ \\
\hline
\end{tabular}

Notes: Data are mean + SEM, $* P=0.007$ significantly less than sham sites.

Abbreviations: BMI, body mass index; USG, urine specific gravity; SEM, standard error of the mean.

are presented in Tables 2 and 3. There were no significant differences across time for room and subject characteristics (room temperature, room humidity, USG, site impedance, $\mathrm{mA}$ sensory, $\mathrm{mA}$ maximum). All subjects met the inclusion criteria for USG prior to each test session.

\section{Impedance greater at SSS than OSS}

In Experiment 2, mean impedance taken with the impedance meter was $13.55 \pm 0.36 \Omega$ for OSS and $14.99 \pm 0.38 \Omega$ for SSS electrode placement sites (Figure 3A). This was significantly different $\left(F_{1,23}=59.30, P=0.0001\right)$ but was not significantly different between visits. Impedance measures through the TENS unit at 3,6, 9, and $12 \mathrm{~mA}$ settings were assessed at each visit and pooled across visits. Calculated impedance ranged from 1.118 to $1.218 \Omega$ for OSS and 1.152 to 1.323 $\Omega$ for SSS and was significantly different $\left(F_{1,23}=10.36\right.$, $P=0.004$ ) (Figure 3B).

\section{TENS increases PPT similarly between SSS and OSS}

When compared to pre-TENS, PPT at the forearm was significantly increased with OSS-TENS $(P=0.002)$, SSSTENS ( $P=0.0001)$, and P-TENS $(P=0.007$ ) (Figure $3 C$ ). The AUC for OSS-TENS was significantly higher than P-TENS $(P=0.024)$. This was also true for SSS-TENS compared to P-TENS $(P=0.025)$. There was no significant difference in the AUC between the two active treatments $(P=0.81)$ (Figure 3D).

\section{TENS amplitude}

The pulse amplitude required to achieve the desired (highest tolerable) TENS treatment intensity was similar between the two active TENS treatments (ie, mean amplitude was 19.2 $\pm 1.0 \mathrm{~mA}$ for OSS-TENS, and $21.2 \pm 0.7 \mathrm{~mA}$ for SSS-TENS). The time course for mean TENS amplitude 


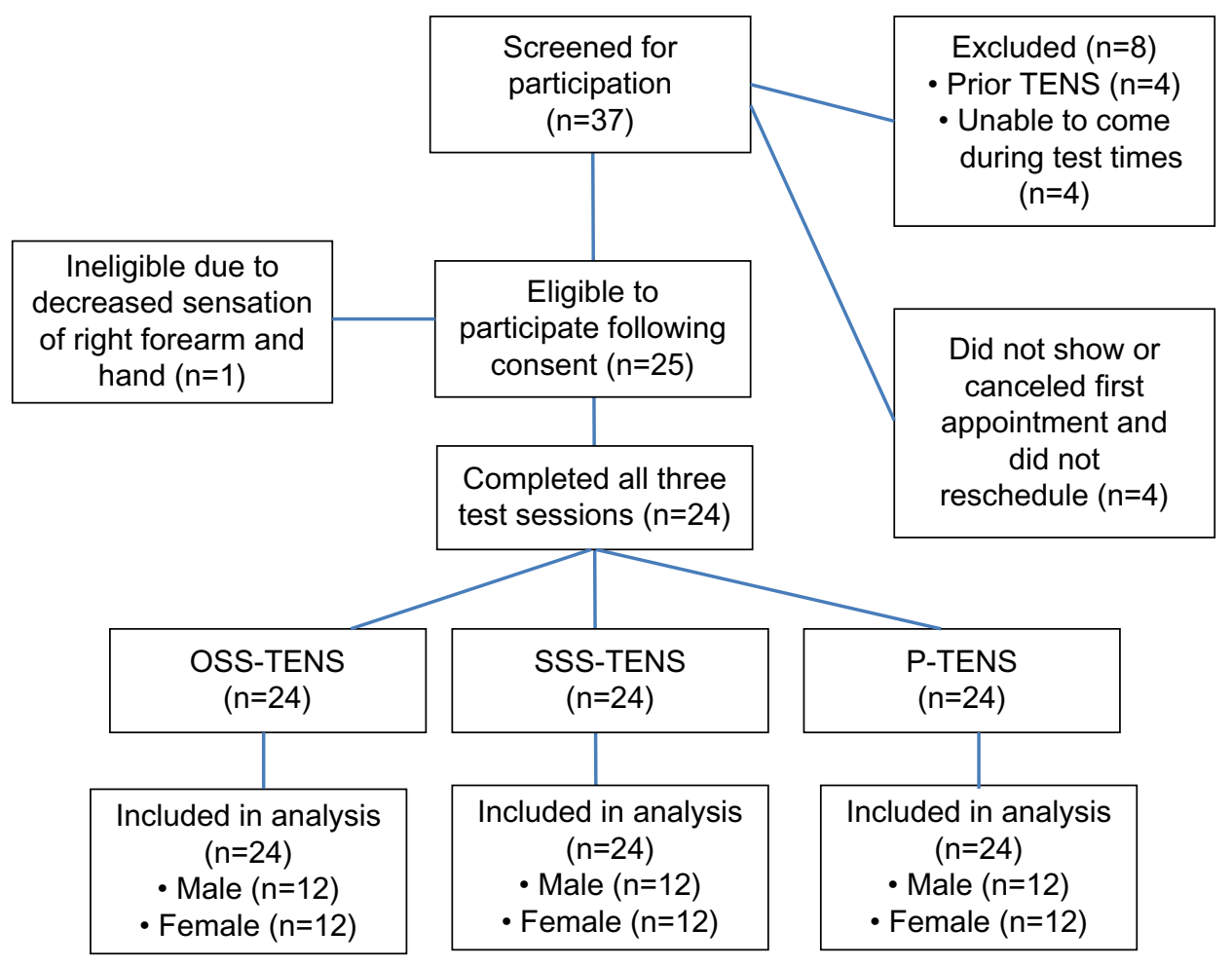

Figure 2 Consort diagram for the TENS experiment in this crossover design.

Abbreviations: TENS, transcutaneous electrical nerve stimulation; OSS, optimal site selection; SSS, sham site selection; P, placebo.

every 5 minutes during the 30 -minute treatment is shown in Figure 4. When subjects received TENS at OSS, 12/24 (50\%) chose treatment amplitudes that achieved motor contraction. When subjects received TENS at SSS, 8/24 (30\%) chose amplitudes that achieved motor contraction.

\section{Perceived comfort}

When subjects received P-TENS, the comfort was rated as $0.41 \pm 0.11$. For the two active TENS treatments, OSS was rated as $2.5 \pm 0.34$ and SSS was rated as $2.1 \pm 0.42$. There were no significant differences between the active TENS treatments $(P=0.20)$. P-TENS comfort scores were significantly lower than both active TENS treatments $(P=0.0001)$.

Table 2 Demographics and baseline measures for Experiment 2 comparing the effects of TENS on optimal and sham sites

\begin{tabular}{lllll}
\hline Variables & Visit I & Visit 2 & Visit 3 & P-value \\
\hline Age & $31.75 \pm 2.19$ & $31.75 \pm 2.19$ & $31.75 \pm 2.19$ & N/A \\
BMI & $25.07 \pm 1.09$ & $25.09 \pm 1.08$ & $25.04 \pm 1.09$ & 0.66 \\
Room & $71.45 \pm 0.39$ & $71.94 \pm 0.36$ & $72.04 \pm 0.4$ & 0.16 \\
temperature $\left({ }^{\circ} \mathrm{F}\right)$ & & & & \\
Room humidity (\%) & $51.25 \pm 0.31$ & $50.9 \pm 0.31$ & $51.04 \pm 0.32$ & 0.57 \\
USG & $1.008 \pm 0.001$ & $1.010 \pm 0.002$ & $1.008 \pm 0.001$ & 0.79 \\
\hline
\end{tabular}

Notes: Demographics/baseline $M=12, F=12$. Data are mean + SEM.

Abbreviations: TENS, transcutaneous electrical nerve stimulation; SEM, standard error of the mean; M, male; F, female; BMI, body mass index; USG, urine specific gravity; N/A, not applicable.

\section{Experimental blinding}

The PPT assessor was blinded to differences between OSS and SSS application $100 \%$ of the time. The assessor was also blinded to whether the subjects received placebo or active TENS $95 \%$ of the time.

\section{Discussion}

The current study showed that skin impedance was significantly lower at OSS when compared to SSS using two independent methods. Despite this difference in skin impedance, there was no difference in PPT produced by placing TENS at OSS and SSS. While both OSS-TENS and SSS-TENS resulted in significantly higher PPTs than OSS-P-TENS, both produced equivalent increases in PPTs. Further, the amplitude of TENS used to achieve a strong but comfortable intensity and subject comfort was similar between the OSS-TENS and SSS-TENS. Thus, TENS delivered over OSS was not more effective than TENS delivered over SSS, suggesting that skin impedance is not a factor in effectiveness of TENS when applied at strong but comfortable intensities.

Previous experiments measuring PPT in healthy controls using both high- and low-frequency stimulation, applied to either the arm or the leg, show that application of EA over acupoints produces greater analgesia when compared to 
Table 3 Primary outcomes measures prior to application of TENS

\begin{tabular}{|c|c|c|c|c|}
\hline Outcome measures - baseline & OSS-TENS & SSS-TENS & P-TENS & $P$-value \\
\hline PPT (kPa) & $191.3 \pm 15.7(\mid 58.8-223.7)$ & $202.4 \pm 19.8(161.4-243.4)$ & $212.7 \pm 25.1(160.8-264.6)$ & 0.22 \\
\hline OSS impedance & $13.2 \pm 0.60(|2.25-| 4.7 \mid)$ & $13.4 \pm 0.55(\mid 2.43-14.92)$ & $13.1 \pm 0.53(\mid 2.08-14.92)$ & 0.77 \\
\hline SSS impedance & $|4.95 \pm 0.7|(\mid 3.6-16.06)$ & $14.30 \pm 0.56(\mid 3.47-15.99)$ & $14.38 \pm 0.54(13.72-17.04)$ & 0.12 \\
\hline $\mathrm{mA}$ sensory threshold & $6.6 \pm 0.48(5.60-7.57)$ & $6.7 \pm 0.29(6.07-7.26)$ & $7 \pm 0.39(6.2-7.8)$ & 0.35 \\
\hline $\mathrm{mA}$ maximum & $19.2 \pm 1.03(17.07-21.34)$ & $21.0 \pm 0.77(19.4 I-22.60)$ & $15.1 \pm 0.68^{\mathrm{a}}(\mid 3.76-16.57)$ & 0.0001 \\
\hline
\end{tabular}

Notes: Data are mean \pm SEM and (95\% confidence intervals). aSignificantly different from OSS-TENS and SSS-TENS.

Abbreviations: TENS, transcutaneous electrical nerve stimulation; SEM, standard error of the mean; CI, confidence interval; OSS, optimal site selection; SSS, sham site selection; P, placebo; PPT, pressure pain threshold.

manual acupuncture or sham acupuncture. ${ }^{19-22}$ However, these studies did not examine the effect of using sham acupuncture sites for treatment. When applying TENS to the proximal forearm and measuring PPT at the hand, similar to the current study, the two active treatments (true versus sham site application) differed significantly from baseline, but not from one another. ${ }^{18}$ In addition, acupoint electrical stimulation in patient populations decreases pain and pruritus, opioid and fentanyl intake, and associated negative side effects in various populations when compared to placebo stimulation. ${ }^{16,23-27}$ Of these reports, two examined true versus sham sites of electrical stimulation application, one with TENS ${ }^{16}$ and another with
A

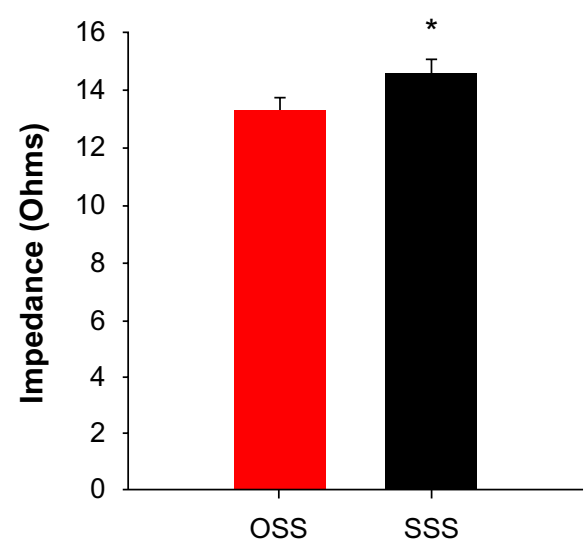

C

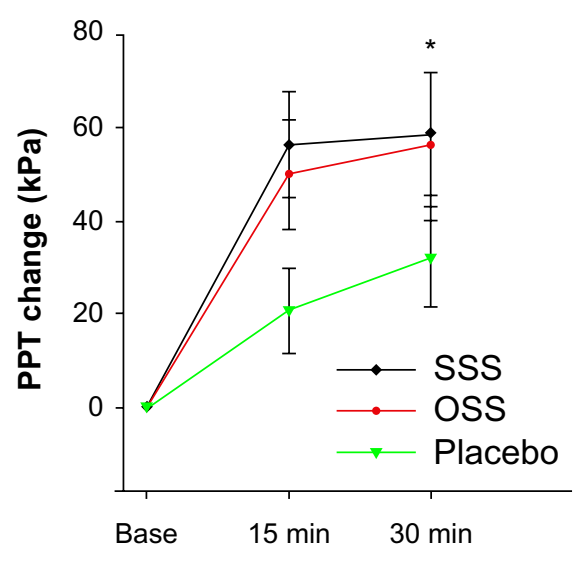

B

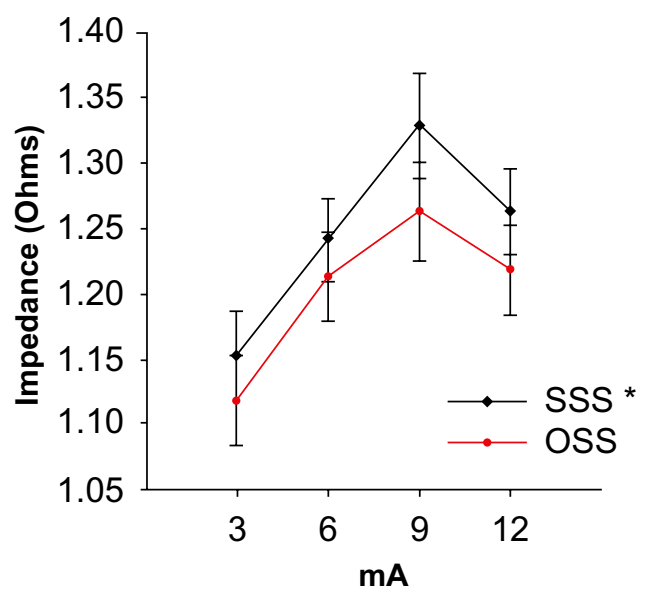

D

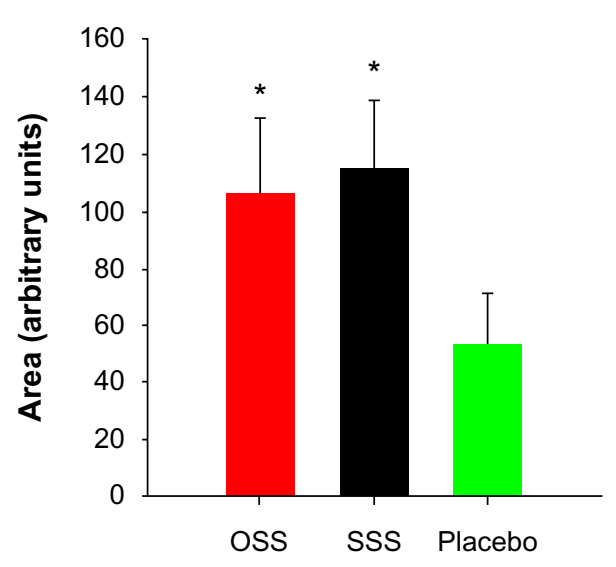

Figure 3 Impedance and pressure pain threshold results.

Notes: (A) Average impedance $(\Omega)$ using an impedance meter with sham sites significantly higher than optimal sites $(* P<0.05)$. (B) Impedance $(\Omega)$ measures using the TENS unit at three amplitudes with sham sites significantly higher than optimal sites $\left({ }^{*} P<0.05\right)$. (C) Change in PPT at 15 and 30 minutes. OSS-TENS and SSS-TENS show significantly greater change in PPT when compared to P-TENS (placebo), yet are not significantly different from each other $(* P<0.05)$. (D) Area under the curve from I5- and 30-minute time points, with both active treatments significantly different from placebo, but not different from one another $(* P<0.05)$.

Abbreviations: OSS, optimal site selection; SSS, sham site selection; PPT, pressure pain threshold; TENS, transcutaneous electrical nerve stimulation; P, placebo; min, minutes. 


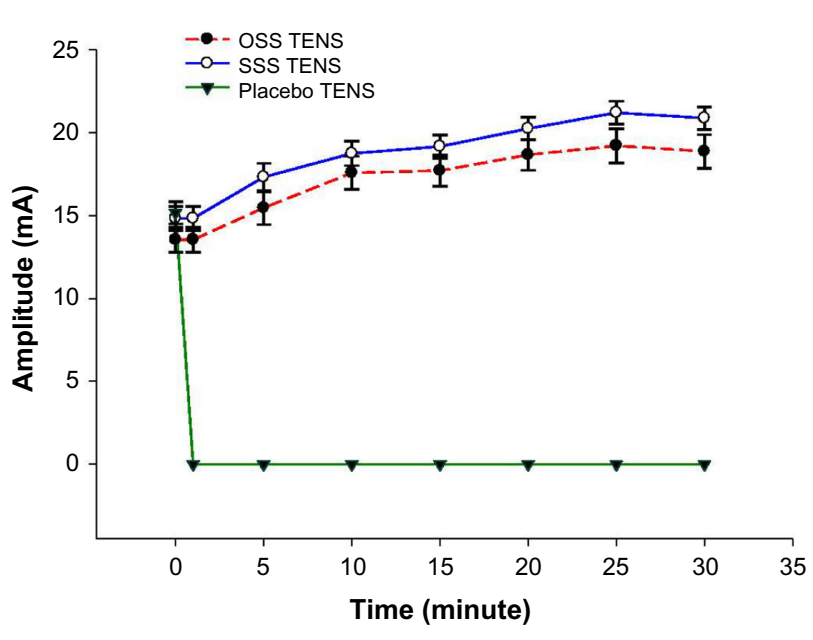

Figure 4 Time course for TENS amplitude.

Note: OSS-TENS and SSS-TENS significantly greater than P-TENS.

Abbreviations: TENS, transcutaneous electrical nerve stimulation; OSS, optimal site selection; SSS, sham site selection; $P$, placebo.

EA. ${ }^{25}$ In both instances, stimulation over acupoints provided greater analgesia. Differences could be related to potential differences in TENS effectiveness between the healthy controls (current study) and the pain population. ${ }^{40}$

Several theories suggest that lower impedance at points on the skin could be due to proximity to peripheral nerve branches. ${ }^{41-43}$ Previously, we have shown that activation of deep tissue afferents is required to produce analgesia, ${ }^{44}$ suggesting that adequate amplitude is required to produce analgesia. Indeed, our prior studies show increasing analgesia with increasing intensities of stimulation, ${ }^{39,45}$ and clinical trials show greater effectiveness when TENS is delivered at adequate intensities compared to lower intensities. ${ }^{46,47}$ Thus, skin impedance may play a minimal role when TENS is given at adequate intensities to produce hypoalgesia. Interestingly, when TENS was applied over acupuncture points in the case of postoperative pain, the stimulation intensity reported was 9-12 $\mathrm{mA}^{16}$ as compared to $19-21 \mathrm{~mA}$ delivered in our study. Figure $3 \mathrm{~B}$ tissue shows that resistance appears to peak at $9 \mathrm{~mA}$. Future studies could be designed to test tissue impedance at higher intensities to determine if resistance continued to decrease as stimulation intensity is increased and to determine if the relationship of sham $>$ optimal resistance remained the same. Establishing these relationships in patients with pain conditions would be of further interest as well.

Interestingly, while there was no difference in PPTs between OSS-TENS and SSS-TENS, there was a difference in the number of subjects where amplitudes produced motor contractions $-50 \%$ of the time over OSS and $30 \%$ of the time over SSS. This could imply that the OSS sites were closer to the motor endplate or nerve fibers allowing earlier depolarization of A-alpha fibers. Further, comfort was rated as similar between OSS-TENS and SSS-TENS, indicating that the presence of motor contractions did not appear to influence perceived comfort. Thus, differences in impedance observed between sites characterized as OSS and SSS may lead to a higher likelihood of producing a motor contraction but does not play a role in effectiveness or comfort.

\section{Blinding of active and P-TENS}

The PPT assessor was blinded to electrode site $100 \%$ of the time and to TENS treatment $95 \%$ of the time by virtue of the randomization protocol, and this was in agreement with prior studies using this P-TENS unit. ${ }^{38,45,48}$ Adequate blinding is important to remove bias in interpretation from the subject and the assessor. Prior TENS studies use separate instructions to the subject for active and P-TENS which can influence patient expectations and have profound effect on treatment outcome. ${ }^{49-53}$ Subjects involved in this study were advised that three different TENS treatments coupled with two different electrode site placements were being evaluated allowing for the same script to be used for each visit. By giving the same instructions, we were able to show that active TENS in healthy normal subjects increased PPT compared to P-TENS. Because each subject received all three treatments, it is possible that their perception was biased with repetitive treatments; however, randomization to treatment order was utilized to decrease an order effect.

\section{Study limitations}

The inferences made from this study can be applied only to the use of TENS in the forearm of healthy subjects without a painful condition. When considering the electrode location and intensities utilized, the volume of tissue in the forearm conducting the electrical charge would be smaller than the instance where electrodes are placed to treat many common clinical conditions. It is also unclear whether impedance is different in patients with acute or chronic pain conditions. Finally, using the TENS unit to assess impedance was a novel application, and we did not monitor impedance at intensities greater than $12 \mathrm{~mA}$.

\section{Conclusion}

In summary, the current randomized trial examines skin impedance differences between sites deemed "optimal" and those categorized as "sham" and examines the effect of electrode site selection when using TENS on the upper limb of healthy adults. Although significant differences in impedance 
were found between the electrode sites, when TENS is applied at an adequate intensity, skin impedance was not a factor in TENS effect on PPT. Further investigation of this parameter should include patients who present with painful conditions to determine if electrode site selection is a critical factor when using TENS to reduce hyperalgesia. Regardless, clinicians should focus on choosing sites that are well tolerated by patients to allow for strong, nonpainful levels of stimulation, which may include motor contractions.

\section{Acknowledgments}

Financial support was provided by The University of Iowa, Carver College of Medicine and NIH R34 planning grant, although neither of these had involvement in the study design, data collection, analysis or interpretation, writing or decision for submission of the report.

We thank Arliss Dudley-Cash, Nicole Englehardt Jennifer Lee, Kristin Hood, and Shannon Lehman for assistance with data collection.

\section{Disclosure}

Dr Kathleen A Sluka is a consultant for DJO Global. The authors report no other conflicts of interest in this work.

\section{References}

1. Dowswell T, Bedwell C, Lavender T, Neilson JP. Transcutaneous electrical nerve stimulation (TENS) for pain relief in labour. Cochrane Database Syst Rev. 2009;(2):CD007214.

2. Hurlow A, Bennett MI, Robb KA, Johnson MI, Simpson KH, Oxberry SG. Transcutaneous electric nerve stimulation (TENS) for cancer pain in adults. Cochrane Database Syst Rev. 2012;3:CD006276.

3. Khadilkar A, Odebiyi DO, Brosseau L, Wells GA. Transcutaneous electrical nerve stimulation (TENS) versus placebo for chronic low-back pain. Cochrane Database Syst Rev. 2008;(4):CD003008.

4. Kroeling P, Gross AR, Goldsmith CH; Cervical Overview Group. A cochrane review of electrotherapy for mechanical neck disorders. Spine (Phila Pa 1976). 2005;30:E641-E648.

5. Mulvey MR, Bagnall AM, Johnson MI, Marchant PR. Transcutaneous electrical nerve stimulation (TENS) for phantom pain and stump pain following amputation in adults. Cochrane Database Syst Rev. 2010;(5): CD007264.

6. Nnoaham KE, Kumbang J. Transcutaneous electrical nerve stimulation (TENS) for chronic pain. Cochrane Database Syst Rev. 2008; (3):CD003222.

7. Rutjes AW, Nuesch E, Sterchi R, et al. Transcutaneous electrostimulation for osteoarthritis of the knee. Cochrane Database Syst Rev. 2009;(4):CD002823.

8. Walsh DM, Howe TE, Johnson MI, Sluka KA. Transcutaneous electrical nerve stimulation for acute pain. Cochrane Database Syst Rev. 2009;(2):CD006142.

9. Hughes N, Bennett MI, Johnson MI. An investigation into the magnitude of the current window and perception of transcutaneous electrical nerve stimulation (TENS) sensation at various frequencies and body sites in healthy human participants. Clin J Pain. 2013;29:146-153.

10. Berlant SR. Method of determining optimal stimulation sites for transcutaneous electrical nerve stimulation. Phys Ther. 1984;64: 924-928.
11. Hecox B, Mehreteab TA, Weisberg J, Sanko J. Integrating Physical Agents in Rehabilitation. Upper Saddle River, NJ: Pearson Education Inc.; 2006.

12. Lamm KE. Optimal Placement Techniques for TENS. 1989. Course booklet from continuing education course. Available from: http://www. kenlammphysicaltherapy.com.

13. Nelson RM, Hayes KW, Currier DP. Clinical Electrotherapy. Stamford, CT: Appleton and Lang; 1999.

14. Prentice WE. Therapeutic Modalities for Allied Health Professionals. New York, NY: McGraw-Hill Health Professions Division; 1998.

15. Robinson AJ, Snyder-Mackler L. Clinical Electrophysiology Electrotherapy and Electrophysical Testing. Baltimore, MD: Williams \& Wilkins; 1995.

16. Chen L, Tang J, White PF, et al. The effect of location of transcutaneous electrical nerve stimulation on postoperative opioid analgesic requirement: acupoint versus nonacupoint stimulation. Anesth Analg. 1998;87: 1129-1134.

17. Wang N, Hui-Chan C. Effects of acupoints TENS on heat pain threshold in normal subjects. Chin Med J (Engl). 2003;116:1864-1868.

18. Cheing GL, Chan WW. Influence of choice of electrical stimulation site on peripheral neurophysiological and hypoalgesic effects. $J$ Rehabil Med. 2009;41:412-417.

19. Barlas P, Ting SL, Chesterton LS, Jones PW, Sim J. Effects of intensity of electroacupuncture upon experimental pain in healthy human volunteers: a randomized, double-blind, placebo-controlled study. Pain. 2006;122: 81-89.

20. Lang PM, Stoer J, Schober GM, Audette JF, Irnich D. Bilateral acupuncture analgesia observed by quantitative sensory testing in healthy volunteers. Anesth Analg. 2010;110:1448-1456.

21. Schliessbach J, van der Klift E, Arendt-Nielsen L, Curatolo M, Streitberger K. The effect of brief electrical and manual acupuncture stimulation on mechanical experimental pain. Pain Med. 2011;12: 268-275.

22. Chen CC, Johnson MI. An investigation into the hypoalgesic effects of high- and low-frequency transcutaneous electrical nerve stimulation (TENS) on experimentally-induced blunt pressure pain in healthy human participants. J Pain. 2010;11:53-61.

23. Liu YY, Duan SE, Cai MX, Zou P, Lai Y, Li YL. Evaluation of transcutaneous electroacupoint stimulation with the train-of-four mode for preventing nausea and vomiting after laparoscopic cholecystectomy. Chin J Integr Med. 2008;14:94-97.

24. Lee SH, Lee BC. Electroacupuncture relieves pain in men with chronic prostatitis/chronic pelvic pain syndrome: three-arm randomized trial. Urology. 2009;73:1036-1041.

25. Ma YX, Ma LX, Liu XL, et al. A comparative study on the immediate effects of electroacupuncture at Sanyinjiao (SP6), Xuanzhong (GB39) and a non-meridian point, on menstrual pain and uterine arterial blood flow, in primary dysmenorrhea patients. Pain Med. 2010;11:1564-1575.

26. Lan F, Ma YH, Xue JX, Wang TL, Ma DQ. Transcutaneous electrical nerve stimulation on acupoints reduces fentanyl requirement for postoperative pain relief after total hip arthroplasty in elderly patients. Minerva Anestesiol. 2012;78:887-895.

27. Wang H, Xie Y, Zhang Q, et al. Transcutaneous electric acupoint stimulation reduce intra-operative remifentanil consumption and alleviates postoperative side-effects in patients undergoing sinusotomy: a prospective, randomized, placebo-controlled trial. Br J Anaesth. 2014;112:1075-1082.

28. Hyvarinen J, Karlsson M. Low-resistance skin points that may acupuncture loci. Med Biol. 1977;55:88-94.

29. Pearson S, Colbert AP, McNames J, Baumgartner M, Hammerschlag R. Electrical skin impedance at acupuncture points. J Altern Complement Med. 2007;13:409-418.

30. Roppel RM, Mitchell F Jr. Skin points of anomalously low electric resistance: current-voltage characteristics and relationships to peripheral stimulation therapies. J Am Osteopath Assoc. 1975;74:877-878.

31. Casa DJ, Armstrong LE, Hillman SK, et al. National athletic trainers' association position statement: fluid replacement for athletes. J Athl Train. 2000;35:212-224. 
32. Pross N, Demazieres A, Girard N, et al. Influence of progressive fluid restriction on mood and physiological markers of dehydration in women. Br J Nutr. 2013;109:313-321.

33. UIHC Department of Pathology Laboratory Services Handbook. Urinalysis. 10/28/2008; 8/6/2009. Available from: http://www. healthcare.uiowa.edu/path_handbook/handbook/test2590.html.

34. Fogari R, Bernardi L, Bardelli R, Casati A, Finardi G. Topographic variations of the skin impedence in the normal young adults. Boll Soc Ital Biol Sper. 1982;58:745-751.

35. Kim MS, Cho YC, Moon JH, Pak SC. A characteristic estimation of bio-signals for electro-acupuncture stimulations in human subjects. $\mathrm{Am}$ J Chin Med. 2009;37:505-517.

36. Doig GS, Simpson F. Randomization and allocation concealment: a practical guide for researchers. J Crit Care. 2005;20:187-191; discussion 191-193.

37. Fischer AA. Reliability of the pressure algometer as a measure of myofascial trigger point sensitivity. Pain. 1987;28:411-414.

38. Rakel B, Cooper N, Adams HJ, et al. A new transient sham TENS device allows for investigator blinding while delivering a true placebo treatment. J Pain. 2010;11:230-238.

39. Pantaleao MA, Laurino MF, Gallego NL, et al. Adjusting pulse amplitude during TENS application produces greater hypoalgesia. J Pain. 2011;12:581-590.

40. Zhang R, Lao L, Ren K, Berman BM. Mechanisms of acupunctureelectroacupuncture on persistent pain. Anesthesiology. 2014;120 482-503.

41. Ahn AC, Martinsen OG. Electrical characterization of acupuncture points: technical issues and challenges. J Altern Complement Med. 2007; $13: 817-824$

42. Martinsen OG, Grimnes S. Facts and myths about electrical measurement of stratum corneum hydration state. Dermatology. 2001;202:87-89.

43. Martinsen OG, Grimnes S, Karlsen J. Electrical methods for skin moisture assessment. Skin Pharmacol. 1995;8:237-245.
44. Radhakrishnan R, Sluka KA. Deep tissue afferents, but not cutaneous afferents, mediate transcutaneous electrical nerve stimulation-induced antihyperalgesia. J Pain. 2005;6:673-680.

45. Moran F, Leonard T, Hawthorne S, et al. Hypoalgesia in response to transcutaneous electrical nerve stimulation (TENS) depends on stimulation intensity. J Pain. 2011;12:929-935.

46. Bjordal JM, Johnson MI, Ljunggreen AE. Transcutaneous electrical nerve stimulation (TENS) can reduce postoperative analgesic consumption. A meta-analysis with assessment of optimal treatment parameters for postoperative pain. Eur J Pain. 2003;7:181-188.

47. Bjordal JM, Johnson MI, Lopes-Martins RA, Bogen B, Chow R, Ljunggren AE. Short-term efficacy of physical interventions in osteoarthritic knee pain. A systematic review and meta-analysis of randomised placebo-controlled trials. BMC Musculoskelet Disord. 2007;8:51.

48. Dailey DL, Rakel BA, Vance CG, et al. Transcutaneous electrical nerve stimulation reduces pain, fatigue and hyperalgesia while restoring central inhibition in primary fibromyalgia. Pain. 2013;154:2554-2562.

49. Bialosky JE, Bishop MD, Robinson ME, Barabas JA, George SZ. The influence of expectation on spinal manipulation induced hypoalgesia: an experimental study in normal subjects. BMC Musculoskelet Disord. 2008;9:19.

50. George SZ, Robinson ME. Dynamic nature of the placebo response. J Orthop Sports Phys Ther. 2010;40:452-454.

51. Levine JD, Gordon NC. Influence of the method of drug administration on analgesic response. Nature. 1984;312:755-756.

52. Levine JD, Gordon NC. Method of administration determines the effect of naloxone on pain. Brain Res. 1986;365:377-378.

53. Marchand S, Charest J, Li J, Chenard JR, Lavignolle B, Laurencelle L. Is TENS purely a placebo effect? A controlled study on chronic low back pain. Pain. 1993;54:99-106.
Journal of Pain Research

\section{Publish your work in this journal}

The Journal of Pain Research is an international, peer-reviewed, open access, online journal that welcomes laboratory and clinical findings in the fields of pain research and the prevention and management of pain. Original research, reviews, symposium reports, hypothesis formation and commentaries are all considered for publication.

\section{Dovepress}

The manuscript management system is completely online and includes a very quick and fair peer-review system, which is all easy to use. Visit http://www.dovepress.com/testimonials.php to read real quotes from published authors. 